\title{
Monothiol glutaredoxins: a common domain for multiple functions
}

\author{
Enrique Herrero* and María Angeles de la Torre-Ruiz
}

Departament de Ciències Mèdiques Bàsiques, Facultat de Medicina, Universitat de Lleida, Montserrat Roig 2, 25008-Lleida, Spain, Phone: +34 973 702409, Fax : +34973 702428,E-mail : enric.herrero@cmb.udl.es

${ }^{*}$ Corresponding author

Running title: Monothiol glutaredoxins and redox regulation 
Abstract. Monothiol glutaredoxins with the CGFS sequence at the active site are widespread among prokaryotes and eukaryotes. Two subclasses exist, those with a single glutaredoxin domain and those with a thioredoxin-like region followed by one or more glutaredoxin domains. Studies in Saccharomyces cerevisiae have demonstrated the role of the Grx5 protein in the biogenesis of iron-sulphur clusters. Grx5 homologues in other eukaryotes could carry out similar functions. Two S. cerevisiae monothiol glutaredoxins with the thioredoxin-like extension, Grx3 and Grx4, are modulators of the transcriptional activator Aft1, which regulates iron uptake in yeast. The human PICOT protein is a Grx3/Grx4 homologue with the same hybrid primary structure, which regulates protein kinase $\mathrm{C}$ activity and may participate in physiological processes such as control of cardiac function. Therefore, monothiol glutaredoxins share a common basic structural motif and biochemical mechanism of action, while participating in a diversity of cellular functions as protein redox regulators.

Keywords. Glutaredoxin, redox regulation, gutathione, oxidative stress, iron-sulphur cluster, transcription regulator, signal transduction. 


\section{Introduction}

Aerobic life generates highly oxidant molecular species as a consequence of incomplete reduction of molecular oxygen to water. These reactive oxygen species (ROS) include superoxide radical, hydroxyl radical and singlet oxygen, besides hydrogen peroxide [1]. They react with proteins, lipids and nucleic acids, modifying them and causing different degrees of toxicity. Oxidative stress may be provoked not only by ROS generation during aerobic respiration and other metabolic processes, but also by external oxidants.

Cysteine is a key amino acid for protein folding, metal coordination and enzyme catalysis, due to the redox properties of its thiol group [2-4]. In parallel, it is especially sensitive to ROS action [5]. ROS modification of the free thiol of the cysteine molecule leads to increasingly oxidized forms, from disulphide bond to sulphenic (-SOH), sulphinic $\left(-\mathrm{SO}_{2} \mathrm{H}\right)$ and sulphonic $\left(-\mathrm{SO}_{3} \mathrm{H}\right)$ acid. Disulphide bonds between protein cysteine residues can be intra or intermolecular, contributing to the tertiary and quaternary structure of proteins respectively. However, in certain conditions formation of a disulphide bond between two cysteine thiols can inactivate the protein due to alteration of the active protein structure or because specific oxidation of the thiol(s) required for protein activity. On the other hand, mixed disulphides can be formed between a cysteine thiol of a protein and a different molecule, such as the L- $\gamma$-glutamylL-cysteinyl-glycine tripeptide glutathione (GSH in its reduced form). This particular reaction is termed glutathionylation. Formation of disulphide bonds or of sulphenic acid derivatives is a reversible process, while formation of sulphinic and sulphonic acid derivatives has been considered as irreversible, that is, it leads to definitive protein inactivation. However, in yeast cells a sulphiredoxine has recently been characterized which is able to reduce sulphinic acid derivatives [6]. Glutathionylation would be a protective mechanism on protein thiols during oxidative conditions, since it can be reversed [7-10]. Cysteine nitrosylation after oxidative stress (with formation of a -SNO 
group) is another way to reversibly protect thiol groups. Nevertheless, modifications in the redox state of protein cysteines can not be contemplated merely as the final consequence of changes in the oxidative conditions inside the cell, but as well as an active process that may regulate cellular functions [7, 8]. For instance, DNA binding activity of transcriptional factors NF-KB or c-Jun is regulated by glutathionylation of cysteine residues [11, 12]. Also, glutathionylation of an essential cysteine of glyceraldehyde 3-phosphate dehydrogenase inactivates the enzyme in the yeast Saccharomyces cerevisiae, and it has been proposed that the glycolytic flux is regulated through this mechanism after oxidative stress [13]. Disulphide bond formation is important for redox sensors of oxidative stress such as the Escherichia coli Hsp33 [14] or OxyR regulators [15].

The redox state of protein cysteine thiols is regulated by the thioredoxin and glutaredoxin systems [16-19]. Thioredoxins are low molecular weight thiol-disulphide oxidoreductases with a conserved CGPC active site motif, which reduce protein disulphides and regenerate the active thioredoxin enzyme using thioredoxin reductase and electrons from NADPH $[16,17]$. In plant chloroplasts, active thioredoxin is regenerated in the light by the photosynthetic electron transfer chain through ferredoxin and ferredoxin-thioredoxin reductase [20]. Glutaredoxins (Grxs) are also thioldisulphide oxidoreductases, but they employ GSH as reducing agent, the system being completed with NADPH and glutathione reductase $[16,18]$ (Figure 1A). Depending on the active site structure, a division has been established between dithiol (CPY/FC motif) and monothiol (CGFS motif) Grxs [18, 21]. In addition, E. coli glutaredoxin 2 (Grx2) has a hybrid structure between a classical dithiol Grx and a glutathione transferase, although the enzyme activity is characteristic of dithiol Grxs [22]. The increasing number of sequenced genomes is revealing the existence of Grxs with a primary structure that deviates from standard dithiol and monothiol Grxs [23-26], pointing to the complexity of the Grx family. Since they were first discovered, dithiol Grxs have been more thoroughly studied $[18,27]$. Here we will focus our attention 
mainly on the growing information that has recently accumulated on monothiol Grx, from a structural and functional point of view.

\section{Classic dithiol Grxs as thiol oxidoreductases}

Dithiol Grxs were first characterized as reductant of ribonucleotide reductase in $E$. coli mutant cells that lacked thioredoxin activity [28]. In the following years, it was revealed the existence of dithiol Grxs in bacteria, viruses and eukaryotes, and the threedimensional structure of a number of them has been determined (reviewed in Ref. 18). These Grxs are low molecular weight proteins (about $10 \mathrm{kDa}$ ) with the CP/YFC motif and a 'thioredoxin fold' molecular structure. The thioredoxin fold domain (also named 'glutaredoxin fold') is present in protein disulphide isomerases, glutathione peroxidases and glutathione transferases (GSTs), besides thioredoxins, Grxs and some other molecules, that is, in proteins related to redox regulation and response to oxidative stress. All these proteins constitute the 'thioredoxin superfamily' [29]. The thioredoxin fold domain is formed by a four or five-stranded $\beta$-sheet flanked by three or more $\alpha$ helices on either side of the $\beta$-sheet. In glutathione transferases, and also in $E$. coli glutaredoxin 2, the thioredoxin fold domain forms the N-terminal moiety of the protein, which is followed by a $\alpha$-helix-rich C-terminal moiety [22, 30].

Biochemical studies on dithiol GRxs were facilitated with the design of an in vitro assay for measuring enzyme activity [31], which employs $\beta$-hydroxyethil disulphide (HED) as substrate. The assay measures the ability of the Grx enzyme for deglutathionylating the mixed disulphide formed between one GSH molecule and one mercaptoethanol moiety from HED. A more recent assay [32] measures the deglutathionylating activity of Grxs through the increase of fluorescence emission by a triptophan residue when a mixed disulphide between an adjacent cysteine and GSH is reduced. In addition, dithiol Grxs display dehydroascorbate reductase (DHAR) activity 
in in vitro assays [33]. Dithiol Grxs can employ two different mechanisms for reducing disulphide bridges (Figure 1B). The dithiol mechanism is shared with thioredoxins and requires both cysteine residues of the active site [33]. The most $\mathrm{N}$-terminal cysteine forms a mixed disulphide between the Grx molecule and the target protein, the second cysteine being required for solving this intermediate dithiol bridge. GSH molecules restore the reduced active state of Grx. The monothiol mechanism of action only requires the most $\mathrm{N}$-terminal cysteine of the active site [33], and is employed for reducing mixed disulphides between $\mathrm{GSH}$ and the target protein (Figure 1B). Concerning the monothiol mechanism, the formation of an intermediate which consists of a mixed disulphide between the Grx molecule and GSH has been experimentally confirmed [32]. A second GSH molecule is required to solve this disulphide. In contrast to the thiol reducing activity in the HED assay, which only requires the most $\mathrm{N}$-terminal cysteine, the DHAR activity requires both cysteine residues of the Grx active site [33].

To date, the functional studies on dithiol Grxs have mainly focused on E. coli, S. cerevisiae and mammalian Grxs, although information is also emerging on the diversity of plant Grxs [23, 25, 34, 35]. Nevertheless, much less is known on the actual targets of Grxs than on the biochemistry and structure of these proteins. Among the three dithiol glutaredoxins of $E$. coli (Grx1, Grx2, Grx3), one or more of them participate in the activation of ribonucleotide reductase and of 3 '-phosphoadenylysulphate (PAPS) reductase, an enzyme required for sulphate assimilation. They also participate in the reduction of both arsenate reductase and the transcriptional regulator OxyR involved in the oxidative stress response [reviewed in Ref. 18 and 36]. Studies with single and multiple mutants in the E. coli grx genes demonstrate the redundant role of the three Grxs in the defense against external oxidants, either directly or through activation of OxyR. Recently, it has been shown that the three E. coli dithiol Grxs can stimulate the insertion of an iron-sulphur (Fe-S) cluster in the bacterial fumarate nitrate reductase regulator (FNR) apoprotein [37]. FNR acts as an oxygen sensor that regulates gene expression in response to oxygen availability, and its Fe-S cluster plays an essential 
role in controlling gene expression. At least in in vitro conditions, Grx1, Grx2 and Grx3 could regulate the reduced state of essential cysteine residues in the FNR apoprotein required for the Fe-S cluster insertion.

S. cerevisiae contains two classical dithiol Grxs (Grx1 and Grx2) [38]. While Grx1 is exclusively located at the cytosol, Grx2 is located at the cytosol and mitochondria; alternative translation initiation from two in-frame ATG sites explains the existence of the two Grx2 isoforms [39]. Studies with the respective mutants [38] have demonstrated the participation of Grx1 and Grx2 in defense against external oxidants such as hydroperoxides or the superoxide generator menadione, although their functions are only partially overlapping at this respect. At least in part, this defensive role against oxidants could be not strictly related to the thiol oxidoreductase activity of the Grx1 and Grx2 molecules but to their characterized glutathione peroxidase activity [40]. In addition, both yeast Grxs are also active as glutathione transferases [41]. A rice dithiol Grx has also been described which possesses GSH-dependent peroxidase activity [42]. The possible relationship between Grx1 and Grx2 and ribonucleotide reductase and PAPS reductase in S. cerevisiae has not been elucidated. However, similarly to E. coli arsenate reductase, the yeast counterpart of this enzyme, Acr2, is a target of Grx1, which deglutathionylates an intermediate mixed disulphide formed between Acr2 and oxidized glutathione formed during the conversion of $\mathrm{As}(\mathrm{V})$ into As(III) [43].

Human cells contain two dithiol Grxs (reviewed in Ref. 19). Grx1 is cytosolic and participates in such diverse functions as ribonucleotide and dehydroascorbate reduction, regulation of transcription factors and apoptosis. Grx2 has two isoforms derived from the same gene by alternative exon usage, and they are respectively targeted to the mitochondria and nucleus $[44,45]$. Grx2 has an unusual motif (CSYC) at the active site, which results in lower activity in the HED assay, although the molecule is effective in the deglutathionylation reaction [45]. Grx2 contains a Fe-S cluster that bridges two Grx2 monomers and acts as a sensor of oxidant conditions 
[46]. The position of the cluster in the Grx2 molecule is controversial; while the original study [46] proposed that it is located independent of the active site, a more recent work [47] indicates that it is coordinated to an active site cysteine. Degradation of the cluster under oxidative stress results in formation of Grx2 monomers and consequent enzyme activation. These observations therefore relate Grx activity with responses to oxidative stress conditions, the Fe-S clusters acting as sensors of these conditions. Poplar Grx C1 has also been described to contain an associated Fe-S cluster. In this case the cluster is coordinated by the first cysteine of the Grx active site, bridging two enzyme subunits along with two cysteines from two GSH molecules [48]. Using a yeast heterologous system that expresses human forms of $\mathrm{Cu} / \mathrm{Zn}$-dependent superoxide dismutase SOD1, recently it has been shown that amyotrophic lateral sclerosisassociated human SOD1 mutants are more easily reduced and destabilized by the $S$. cerevisiae dithiol Grxs that wild type SOD1 [49]. This observation suggests that a correct balance of Grx activity is needed for controlling SOD1 stability and activity in vivo.

Summarizing, although the possibility exists that dithiol Grxs are general protein redox regulators, increasing evidence supports the idea of more specialized functions for these molecules. These functions include reduction of a diversity of enzymes with unrelated biological functions (such as DNA replication or sulphate assimilation), sensing oxidant conditions, regulation of protein structure, or ROS elimination, among others.

\section{Monothiol Grxs: structurally similar but biochemically different to dithiol Grxs}

Analysis of the sequence of the $S$. cerevisiae genome revealed the existence of three open reading frames (ORFs) whose putative products shared significant homology with S. cerevisiae Grx1 and Grx2 [21]. This homology was higher at the C-terminal moiety of the proteins, while in the three putative molecules the CPY/FC active site signature 
of dithiol Grxs was substituted by the conserved sequence CGFS. Based on that homology, in this initial study the three proteins were named Grx3, Grx4 and Grx5, and they were classified as monothiol Grxs due to the presence of a single cysteine residue at the conserved putative active site [21]. BLAST analysis of completed genomes revealed the existence of homologues of the three yeast monothiol Grxs in bacteria (in particular in cyanobacteria and proteobacteria), protozoa, fungi, plants and animals, that is, they were widespread along the evolutionary scale [23, 25, 35, 50, 51]. Primary sequence comparison denotes a complex structural pattern for monothiol Grxs (Figure 2). Thus, while S. cerevisiae Grx5 contains a single Grx domain, Grx3 and Grx4 have an additional thioredoxin (Trx)-like domain as an N-terminal extension of the Grx domain [50-52]. Both domains are connected by a non-conserved linker region. The Trx-like domain of Grx3 and Grx4 contains a WAD/EPCK sequence that is reminiscent of the authentic thioredoxin active site motif WCGPCK [53]. While all bacterial monothiol Grxs detected to date are of the single Grx domain type, in eukaryotes the Grx-type and the hybrid Trx-Grx type monothiol Grxs coexist [51]. This is the case of human cells (Figure 2), which contain the hGRX5 protein with a single Grx domain [54] and the so called PICOT protein (for PKC-interacting cousin of thioredoxin, see below) [55] with a Trx-Grx structure. It is for this reason that the monothiol Grx domain has also been named as PICOT domain [55]. In fact, the human PICOT protein contains two Grx domains in tandem plus the N-terminal Trx-like domain (Figure 2). This same complex pattern is present in plant monothiol Grxs with the CGFS motif [23, 25, 34, 35]. For instance, four different monothiol (CGFS) Grxs coexist in Arabidopsis thaliana, and one of these, AtGRX3, has three Grx domains in addition to the Trx-like domain (Figure 2).

Only one monothiol Grx three-dimensional structure has been determined to date, that of E. coli Grx4 [56]. This molecule has a single Grx domain, as occurs with $S$. cerevisiae Grx5 (Figure 2). High resolution NMR of the bacterial Grx4 revealed a thioredoxin-fold molecular structure, similar to dithiol Grx. The three-dimensional 
pattern of Grx4 confirmed that of the three cysteine residues in the molecule, only the one in the CGFS motif could interact with GSH, therefore supporting the active site role of this motif [56]. The conservation of important amino acids of the E. coli Grx4 structure in other monothiol Grxs suggests that the Grx4 three-dimensional structure is representative of most monothiol Grxs. Besides the active site differences, other subtle differences exist between the spatial structures of dithiol and monothiol Grxs, which affect GSH binding [56]. For instance, although the Grx residues important for interaction with the C-terminal glycine of the GSH molecule are conserved between dithiol and monothiol Grxs, this conservation does not occur in the case of the charged residues of the dithiol Grxs which are responsible of the interaction with the $\alpha$-glutamyl residue of $\mathrm{GSH}$.

The small but significant differences in GSH binding between dithiol and monothiol Grxs may explain the biochemical differences between both types of molecules. In fact, several monothiol Grxs have been purified from extracts of recombinant E. coli cells (Grx4 of E. coli [57], Grx5 of S. cerevisiae [58] and GLP-1 of Plasmodium falciparum [59]), and none of them is active in the HED assay, contrary to all dithiol Grxs tested. This means that monothiol Grxs are unable to deglutathionylate the small mixed disulphide between $\beta$-mercaptoethanol and a GS-moiety. The null activity of monothiol Grxs in the assay could be due to the null $[57,59]$ or low [58] affinity of the oxidized Grxs for GSH in in vitro studies, which correlates with the above mentioned structural characteristics of the monothiol Grx molecules. Initially, a monothiol mechanism of action (Figure $1 B$ ) could be hypothesized to explain the biochemical role of monothiol Grxs as deglutathionylating enzymes [60]. In fact, $S$. cerevisiae Grx5 is able to efficiently reduce rat carbonic anhydrase III in vitro, and its redox potential $(-175 \mathrm{mV})$ places $\mathrm{Grx} 5$ at an intermediate position among thioldisulphide oxidoreductases [58]. An open question is the nature of the molecule physiologically acting as reductant agent for the Grx. The low or null activity of GSH with the monothiol Grxs discards it as hydrogen donor. Recently, it has been shown 
that $E$. coli Grx4 can be reduced in vitro by the bacterial thioredoxin reductase [57], and yeast Grx5 can also be reduced by $E$. coli thioredoxin reductase [58]. It is therefore possible an interplay between the thioredoxin and monothiol Grx systems through this reductase, at least in bacteria.

Another aspect to be solved concerning the mechanism of the monothiol Grx reactions is the possible role of a second cysteine residue in the Grx molecule. Besides the cysteine in the CGFS motif, E. coli Grx4, S. cerevisiae Grx5 and also other (but not all) monothiol Grxs, posses another cysteine residue at a conserved position in the Cterminal part of the molecule (Figure 2). In Grx5, the in vitro deglutathiolytation reaction begins with the formation of a mixed disulphide between the active site cysteine (Cys60) and $\mathrm{GSH}$, and results in the formation of a disulphide bond between Cys60 and the second cysteine (Cys117) [58]. Consequently, a mutant Grx5 lacking Cys117 is defective in the deglutathionylation reaction. Formation of the disulphide bond between both cysteines has also been observed in E. coli Grx4 [57]. Nevertheless, the physiological role of the $\mathrm{C}$-terminal cysteine residue is not clear, as it is not required for the biological function of Grx5 in vivo (see below) [50].

\section{Diverse subcellular locations of monothiol Grxs}

Subcellular localization of the three monothiol Grxs of $S$. cerevisiae has been thoroughly studied. Comparison of the whole product of the GRX5 ORF with the mature protein expressed in yeast cells revealed an $\mathrm{N}$-terminal 28 amino acids-length region acting as a putative mitochondrial targeting sequence. Subsequent cellular subfractionation analyses confirmed that Grx5 localizes at the mitochondrial matrix and that a mutant lacking the $\mathrm{N}$-terminal targeting region remains at the cytosol 61]. Interestingly, this cytosolic version of Grx5 did not rescue the phenotypic defects of a null grx5 mutant (see below), demonstrating that $S$. cerevisiae Grx5 carry out its physiological functions exclusively at the mitochondria [61]. The Schizosaccharomyces 
pombe homologue of Grx5 also localizes at the mitochondria, as determined microscopically with a GFP-tagged version of the protein [62]. The completely sequenced genomes of eleven additional fungal species also contain an ORF coding for a mitochondrially-located Grx5 homologue of the single Grx-domain type, as predicted by in silico analysis [26]. The GLP-1 protein of $P$. falciparum is also predicted to be mitochondrial, with a hypothetical mitochondrial targeting sequence cleaved at position 43 [59]. Therefore, the existence of a mitochondrially-localized monothiol Grx with a single Grx domain could be a general rule among unicellular eukaryotes. The presence of Grx5 homologues in cellular organelles may also be common in plants. Thus, A. thaliana AtGRXcp localizes at chloroplasts and plastids, as experimentally determined [63], while AtGRX4 protein from the same species is predicted to be mitochondrial [23]. Both proteins contain a single Grx domain, with a 63-amino acids signal peptide in the case of AtGRXcp (Figure 2). Another genome that has been thoroughly studied in silico after genome sequencing completion, that of Populus trichocarpa, contains two single Grx domain homologues of Grx5, respectively predicted to be mitochondrial and plastidic [25].

The two S. cerevisiae monothiol Grx with a hybrid Trx-Grx structure (Grx3 and Grx4) are both localized at the nucleus $[52,64]$. At least in the case of Grx3, this nuclear location is constitutive, not being affected by application of external stresses [52]. None consensus nuclear location signal is predicted in the Grx3 sequence, although it is the Trx-like region of the molecule which is responsible for nuclear targeting [52]. Constitutivity of Grx3 nuclear location contrasts which its homologue the human PICOT protein, which translocates from the cytosol to the nucleus in response to oxidative stress [65]. In S. pombe, the only monothiol Grx with a Trx-Grx structure, Grx4, also has a nuclear localization [62]. S. pombe cells have a third monothiol Grx with an atypical CPYS active site, named Grx3, which localizes at the nuclear rim and endoplasmic reticulum [62]. Other fungal [24] and plant species [23, 25] also contain homologues of S. cerevisiae Grx3/Grx4 with the Trx-Grx hybrid structure. For none of 
them the subcellular location has been experimentally determined, and the significance of in silico prediction for nuclear location of proteins is lower than for mitochondrial/plastidic proteins. However, the experimental observations in yeast and human cells suggest that monothiol Grxs may exist in different eukaryotic groups with a constitutive or stress-induced nuclear location. An open question is whether constitutive cytosolic monothiol Grxs also exist.

\section{Monothiol Grxs and Fe-S cluster synthesis}

Techniques are available for easily disrupting S. cerevisiae genes and consequently analyzing of the phenotype of the resulting mutants. This explains why this yeast species is an extremely useful eukaryotic model for functional analysis of genes and their protein products [66]. The determination of the role of S. cerevisiae Grx5 in the formation of Fe-S clusters $[21,61]$ illustrates the above assertion. A null grx5 mutant is hypersensitive to external oxidants and shows protein hypercarbonylation compared to wild type cells, an indication of intracellular oxidative conditions above normal levels [21]. In addition, grx5 mutant cells grow deficiently in synthetic minimal medium. This latter phenotype was taken as a selective condition to isolate genes that suppressed the growth deficiency when overexpressed [61]. As a result, two genes involved in the synthesis of Fe-S clusters, SSQ1 and ISA2, were isolated as suppressors. Both Ssq1 and Isa2 proteins, as Grx5, are located at the mitochondrial matrix [67]. The role of Grx5 in the biogenesis of the Fe-S clusters at the mitochondria was confirmed through the use of regulatable promoters expressing GRX5. Thus, switching-off expression of the gene led to depletion of Grx5 and inactivation of enzymes containing Fe-S clusters such as aconitase and succinate dehydrogenase, with the concomitant accumulation of iron inside the cell [61]. The fact that biosynthesis of leucine, lysine and glutamic acid requires Fe-S enzymes explains the auxotrophic requirement of the grx5 mutant and the growth defects in minimal medium. Characterization of selectable phenotypes for 
the Grx5 function also allowed determining that Cys60 in the CGFS motif, but not Cys117, is essential for the biological activity of the protein [50]. This therefore establishes a discordance between in vivo data and those obtained in vitro (see above) which pointed to a role of Oys117 in the deglutathionylation activity of the enzyme. Maybe in the rather oxidant conditions of the mitochondrial matrix only Cys60 is required for Grx5 activity. In the absence of Grx5, a number of proteins became specifically glutathionylated, among them glyceraldehide 3-phosphate dehydrogenase [68], probably because these same oxidant conditions.

Iron accumulation is characteristic of mutants in other yeast genes involved in the formation of the Fe-S clusters. This accumulation occurs mainly at the mitochondria [67, 69-71]. The disruption of iron homeostasis in Fe-S biogenesis mutants could be explained based on the basis of the participation of a mitochondrial Fe-S protein as a sensor of iron content for the activation of the iron-uptake system. In fact, a mitochondrial iron signal has been shown to participate in cellular iron sensing [72], although the molecular nature of such signal is still not known. The iron-uptake system depends on the transcriptional activators Aft1 and Aft2 and is activated in conditions of internal iron depletion [73-76]. In accordance with this, the grx5 mutant and other mutants in Fe-S cluster biogenesis have constitutively upregulated the Aft1/Aft2dependent iron regulon [77-79]. The hyperoxidation of cell proteins and sensitivity to external oxidants in the absence of Grx5 could be a direct consequence of ironmediated generation of ROS through the Fenton reaction. However, recently it has been observed that in a grx5 mutant high levels of iron can displace manganese in the Mn-dependent Sod2 superoxide dismutase and therefore inactivate this mitochondrial enzyme $[80,81]$. This may also help to explain the oxidant hypersensitivity phenotype of the mutant. Nevertheless, the synthetic lethality of the grx5 and grx2 mutations on one hand and of the grx5 mutation with the grx3 grx4 combination on the other [21] points to a more complex relationship between monothiol and dithiol Grxs in $S$. cerevisiae cells, maybe related to the sensitivity to oxidative stress (see below). 
Synthesis of Fe-S clusters exclusively occurs at the mitochondrial matrix in yeast cells, independently of the final destination of the Fe-S clusters to mitochondrial, nuclear or cytosolic proteins [reviewed in Ref. 67 and 69-71]. However, specific proteins (the CIA machinery) are additionally required to assemble Fe-S clusters into apoproteins destined to the cytosol or the nucleus, once the clusters have been exported from the mitochondria [69-71]. Whether the Fe-S cluster biogenesis is exclusively mitochondrial in higher eukaryotes is still a matter of debate, although the participating components are evolutionary conserved. Bacteria have three Fe-S biosynthetic systems, and one of them (the Isc system) is the equivalent of the mitochondrial biosynthetic system [4]. In yeast cells, the Fe-S clusters are assembled on a scaffold formed by the Isu1/Isu2 protein, using suphur liberated from cysteine by the cysteine desulphurase activity of Nfs1 and iron imported from the cytosol [82]. By analogy to the bacterial Isc system, Isa1 and Isa2 could recruit iron to the Isu1/Isu2 scaffold B3], although it has been proposed that Yfh1 (homologue of the human frataxin protein) could also contribute to the process [67, 82]. Once assembled, the clusters are transferred to the target apoproteins at the mitochondrial matrix. Grx5 participates at these latter steps, together with the Hsp70 chaperone Ssq1 and its cochaperone Jac1 [82]. This picture is however complicated by the fact that two-hybrid analyses show an in vivo interaction between Grx5 and Isa1 [51], and by in silico modeling of the Fe-S assembly process which suggests a participation of Grx5 at the initial assembly steps on the Isu1/Isu2 scaffold [84]. Independently of the step where Grx5 is acting, efforts are required to characterize the exact function of a thiol oxidoreductase such as Grx5 in the process. Protein cysteine residues involved both in the biogenesis of the clusters on the Isu1/Isu2 scaffold and in the transfer of clusters to apoproteins require an appropriate redox state. By acting as a deglutathionylating enzyme, Grx5 could repair mixed disulphides formed between GSH and components of the Fe-S biosynthetic complex under the oxidant conditions of the mitochondrial matrix. 
The above mentioned mitochondrial sensor of iron content in S. cerevisiae cells [72] requires the entire mitochondrial machinery for Fe-S biogenesis, as well as the Atm1 Fe-S transporter localized in the mitochondrial inner membrane. However, the cytosolic CIA machinery is not required for iron sensing [72]. This initially rules out the participation of a conventional cytosolic (or nuclear) Fe-S protein in transducing the iron signal and regulating the activity of Aft1/Aft2.

How conserved is the role of Grx5 along evolution? The homologues in other fungi are predicted also to be mitochondrial [26]. In the case of $S$. pombe, the grx5 mutant grows slower than wild type cells, is hypersensitive to external oxidants and there is a synthetic lethality between the grx5 and grx2 mutations [62], as occurs in $S$. cerevisiae. This makes probable that $S$. pombe Grx5 also participates in the formation of Fe-S clusters. However, some differences exist between the two yeast species. Expression of $S$. cerevisiae GRX5 and $S$. pombe grx $5^{+}$genes is maximal at exponential phase [21, 85], but while GRX5 expresses constitutively and is not induced by external stresses [21], expression of fission yeast $g r x 5^{+}$is induced by osmotic and nitrosative stresses, in a process dependent on the transcription factor Pap1 [85]. Differences between $S$. cerevisiae and $S$. pombe may extend to the whole process of Fe-S cluster synthesis and thiol oxidoreductases requirements. Thus, contrary to $S$. cerevisiae the fission yeast requires mitochondrial forms of glutathione reductase or thioredoxin for the maintenance of Fe-S clusters both in mitochondrial and nonmitochondrial holoproteins [86]. However, the direct participation of those two types of enzymes in the biogenesis of the clusters has not been demonstrated.

The $S$. cerevisiae grx5 mutant has been employed as a tool to analyze the functional conservation of monothiol Grxs. Thus, derivatives of nuclear Grx3 and Grx4 S. cerevisiae proteins have been engineered to target them to the mitochondria of Grx5-deficient cells, using the mitochondrial targeting sequence of the own Grx5 protein. In these conditions, the two Trx-Grx-type proteins Grx3/Grx4 rescue the defects of the grx5 mutant, that is, they are able to participate in the biogenesis of the 
Fe-S clusters [52]. This is not the case for dithiol Grx2. Although the biological function of Grx3 and Grx4 is totally different to Grx5 (see below), the above observation shows that yeast monothiol Grxs can substitute each other provided that compartmental barriers are eliminated. Using the same strategy, it has been demonstrated that two bacterial monothiol Grxs (E. coli Grx4 and Synechocystis sp. GrxC) can also partially substitute for the biological function of Grx5 in S. cerevisiae mitochondria [54], which could be taken as a prove for functional conservation. In the case of E. coli Grx4, a mutant lacking this protein is non-viable $[57,87]$. This points to additional functions for Grx4 besides its possible participation in the formation of Fe-S clusters, since E. coli mutants in most of the genes of the Isc system are viable [4]. Levels of E. coli Grx4 are maximal at stationary phase [57]. However, the fact that Grx4 is upregulated upon iron depletion suggests that the protein may be involved in iron-dependent functions.

With respect to higher eukaryotes, the same experimental approach as above [54] demonstrated that the human (hGRX5) and chicken (cGRX5) homologues also are able to participate in the formation of Fe-S clusters once internalized into $\mathrm{S}$. cerevisiae mitochondria. The gene coding for cGRX5 expresses at diverse tissues in chicken embryos [53]. A thorough study has been carried out by Wingert et al. [88] to demonstrate that the deficiency of Grx5 in zebrafish embryos causes hypochromic anaemia in shiraz mutants, due to inability for assembling Fe-S clusters and consequent inhibition of haem synthesis. The shiraz mutant phenotype was reproduced in wild type embryos injected with grx5 antisense RNA, and this phenotype was rescued by overexpressing $S$. cerevisiae, mouse or human RNAs coding for the respective Grx5 proteins [88]. The study also showed that the GRX5 gene is expressed in mouse embryos, and therefore confirmed the conservation of Grx5 function in the assembly of Fe-S clusters in eukaryotes and how this relates to regulation of haem synthesis at least in the zebrafish vertebrate model. A recent work in $A$. thaliana [63] indicates that the Grx5 homologue in this plant, AtGRXcp, also suppresses the phenotypes of a $S$. cerevisiae grx5 mutant once targeted to the yeast mitochondria. At 
the plant, the AtGRXcp gene expresses in young cotyledons and green leaves, and atgrxcp mutant seedlings are hypersensitive to external oxidants. This, together with the fact that AtCRXcp protein localizes at mitochondria and plastids [63], also supports a connection between monothiol Grxs, syntesis of Fe-S clusters and oxidative stress in plant cells.

\section{Monothiol Grxs and regulation of transcription factors}

The cellular function of both Grx3 and Grx4 monothiol glutaredoxins of S. cerevisiae has remained unknown until very recently. Their nuclear location suggested a function as regulators of nuclear proteins and consequently of gene expression. In accordance with this, Pujol et al. [89] have recently demonstrated that both Grx3 and Grx4 regulate Aft1 nuclear localization, which implies that both glutaredoxins share a function related to the regulation of the cellular iron homeostasis. The Aft1 transcription factor which regulates the expression of the high-affinity iron-uptake regulon (see above) translocates to the nucleus in conditions of iron depletion [90]. Importantly, Aft1 transcription activity is precluded by its nuclear localisation and not by iron availability. In this respect, Grx3 and Grx4 primary function appears to be the regulation of Aft1 nuclear localisation. Grx3 and Grx4 both interact with Aft1 in the nucleus [89, 91]. More specifically, Aft1, Grx3 and Grx4 interact physically in the nucleus forming a functional complex [89]. Each monothiol glutaredoxin interacts with Aft1 in an independent manner, suggesting that Grx3 and Grx4 can function independently in the Aft1 regulation. Interestingly, Aft1 binds to each of Grx and Trx domains of each of both glutaredoxins; however, there exist evidence indicating that only the Grx domain of each of both glutaredoxins drives Aft1 nucleo-cytoplasm translocation [89]. Which is the biological meaning of Aft1 binding to each of both thioredoxin domains? This is a question that deserves further studies in order to offer a deeper insight on Aft1 cellular regulation. 
Both single grx3 and grx4 mutants do not present a very significant and marked phenotype, besides the upregulation of the Aft1 regulon [89, 91]. However, the grx3 grx4 double mutant shows a marked defect in cell cycle progression, intracellular iron accumulation and a clear sensitivity to specific oxidant agents, such as hydroperoxides [89]. Therefore, Grx3 and Grx4 have a redundant and additive function in cell growth and oxidative stress protection. In fact, the absence of both Grx3 and Grx4 function constitute a model for endogenous oxidative stress caused, at least partly, by iron homeostasis missregulation.

On despite of the information available concerning Grx3 and Grx4 function in $S$. cerevisiae, there are still a number of questions which remains unanswered, such as: is there another function for Grx3 and Grx4, and more specifically another function in the oxidative stress defence other than Aft1 regulation?, and which is the biochemical mechanism that Grx3 and Grx4 use to regulate Aft1?.

The $S$. pombe Grx4 protein is the homologue of $S$. cerevisiae Grx3/Grx4 proteins and, as noted above, also has a nuclear location [62]. The target(s) of fission yeast Grx4 remains still unknown, although $g r x 4^{+}$is an essential gene in S. pombe, contrary to $S$. cerevisiae $G R X 3$ and GRX4. This, however, could be explained by the functional redundancy observed for both Grx3 and Grx4 in budding yeast. In the case of $S$. pombe, grx $4^{+}$single gene might account for all the regulatory function exerted in the nucleus. There are consistent similarities among the Trx-Grx type monothiol glutaredoxins of budding and fission yeast. The $g r \times 4^{+}$product is likely to function as monothiol glutaredoxin in fission yeast and is involved in the oxidative protective defence against oxidative stress [85, 95], similarly to Grx3 and Grx4 in S. cerevisiae. It will be interesting to elucidate whether regulation of iron homeostasis and consequently of oxidative endogenous stress would be a characteristic function of monothiol glutaredoxins conserved throughout evolution.

The presence of monothiol glutaredoxins in the nucleus suggests an essential function as regulators of the redox state of nuclear proteins. This raises the question of 
which factor or factors regulate the activity of these nuclear monothiol proteins. In this respect, Grx4 interacts in the nucleus with the $S$. cerevisiae protein kinase piD261/Bud32 [64]. This is a protein that has been related to such diverse functions as bud-site selection, telomere uncapping and elongation or modulation of transcription factors [92-94]. However, a link between $S$. cerevisiae Grx3/Grx4 and these functions has not been established. Bud32 phosphorylates Grx4, what suggests that the kinase might act as a regulator of Grx4 activity [64]. To date, the information about the regulatory proteins which modulate nuclear monothiol glutaredoxins in different organisms is scarce and needs to be investigated further.

\section{Monothiol Grxs and signal transduction}

Thioredoxins and dithiol Grxs can act as redox regulators of signal transduction pathways [8-10]. The human PICOT protein of the complex Trx-Grx type (see above and Figure 2) is also a modulator of signal transduction. Initial studies detected its expression in $\mathrm{T}$ lymphocytes, where it acts as negative regulator of protein kinase $\mathrm{C}$ $\theta$ (PKC $\theta)$ and plays an important role in T cell-receptor-induced activation [96]. After being translocated into the nucleus upon a stress [65], the PICOT protein interacts with PKC $\theta$ through the Trx-like domain and inactivates the kinase activity [96]. This transiently inhibits several PKC targets such as the c-Jun N-terminal kinase and the transcription factors $\mathrm{AP}-1$ and $\mathrm{NF}-\mathrm{\kappa B}$.

The modulator role of PICOT is not restricted to T cells. Thus, PICOT may act as an endogenous negative regulator of cardiac hypertrophy through its PKC inhibitory activity [97]. Transgenic mice cardiomyocites overexpressing PICOT displayed enhanced contractility associated with increased myofilament $\mathrm{Ca}^{2+}$ contractility. These transgenic mice exhibited increased ventricular function and decreased cardiac hypertrophy induced by pressure overload [97]. Therefore, PICOT might regulate PKC- 
mediated signaling pathways participating in the development of cardiac hypertrophy [98].

Yeast PKC participates in very different functions from mammalian PKC proteins, mainly in cell wall remodeling and cellular morphogenesis [99]. However, the above observations with mammalian PICOT proteins make us considering the possibility that the yeast homologues Grx3 and Grx4 might be also regulators of signal transduction pathways besides their role modulating Aft1 activity.

\section{Other proteins acting as monothiol Grxs}

The spectrum of thiol oxidoreductases acting through a monothiol mechanism is still more complex. Fungal cells have ORFs coding for Grx-like proteins with the putative active site sequence CPYS, which contain a $\mathrm{N}$-terminal positioned transmembrane domain [26]. S. pombe Grx3 protein, which locates at the nuclear rim and endoplasmic reticulum, would be an example of such group of Grx-like proteins [62]. S. cerevisiae has two ORFs (YBR024c and YDL010w) coding for S. pombe Grx3 homologues [26], and purified forms of both protein products in fact display thiol oxidoreductase activity in the HED assay. This activity is dependent on the cysteine residue in the CPYS motif (our unpublished observations). These fungal Grx-like proteins therefore constitute a new class of monothiol Grxs that biochemically differ from the CGFS monothiol Grxs, and whose biological function as possible membrane-associated Grxs is unknown.

Most GSTs act as detoxifiers of xenobiotics by conjugating a GSH molecule to the target molecule [30]. This nucleophilic attack by GSH requires a serine or tyrosine residue at the active site of the GST protein [30, 100]. However, omega-class GSTs differ from most other GSTs by having a cysteine residue at the active site which is essential for the enzyme activity [101, 102]. Purified omega-class GSTs from different origins do not have significant activity on standard GST substrates but instead, they are active as thiol oxidoreductases in the HED assay [101-103]. S. cerevisiae contains 
three omega GSTs, named Gto1, Gto2 and Gto3 [103]. Biochemical studies with Gto2 have shown that a single cysteine in the active site is sufficient for the GSH-dependent thiol oxidoreductase activity, indicating that the enzyme acts through a monothiol mechanism [103]. Substitution of the Gto2 active site motif by the active site of Grx5 eliminates the enzyme activity on HED, while replacement of the Grx5 CGFS motif by the Gto2 active site restores some Grx5 activity on HED [103]. These observations emphasize the importance of the CGFS motif for the biochemical properties of monothiol glutaredoxins.

Of the three Gto proteins of $S$. cerevisiae, the biological function of Gto1 has been studied [104]. It is a peroxisomal GST (in contrast to cytosolic Gto2 and Gto3), whose absence causes dysfunction in the biosynthetic pathway of sulfur amino acids. It has been proposed [104] that Gto1 would be a redox regulator of cystathionine $\beta$-lyase, another peroxisomal protein required for transulphuration from cysteine to homocysteine in yeast cells. S. cerevisiae Gto1 therefore exemplifies an enzyme with monothiol Grx activity with a different location from other monothiol Grxs described to date. However, the peroxisome location of Gto1 is probably a recent evolutionary acquisition in yeast species closely related to $S$. cerevisiae $[104,105]$, and no examples of peroxisomal omega-class GSTs exist in higher eukaryotes. This fact would reflect the evolutionary plasticity of peroxisomes [105].

\section{Conclusions}

Monothiol Grxs with the CGFS active site share a common structural domain and probably also a common mechanism of enzyme action. The presence of an additional thioredoxin-like domain in some of them may be relevant for expanding the number of cellular compartments where they can be localized, but also for substrate specificity. The diversity of cellular compartments where monothiol Grxs carry out their activities and of protein targets help to explain the broad spectrum of cellular functions in which 
they participate through their glutathionylating activity. These include functions as diverse as synthesis of Fe-S clusters, activation of transcription factors or modulation of signal transduction pathways. While there is increasing evidence that participation of monothiol Grxs with a single Grx domain in Fe-S cluster biogenesis is evolutionary conserved, at present there are not data supporting conservation of other specific functions. However, the diversity of organisms, both prokaryotic and eukaryotic, that contain different subclasses of monothiol Grxs point to important biological functions displayed by these thiol oxidoreductases, related to protein redox regulation and defense against oxidative stress.

Acknowledgments. Recent work carried out by the authors was supported by grants BFU2004-03167 (Ministerio de Educación y Ciencia), PI030734 (Fondo de Investigaciones Sanitarias) and 2005SGR00677 (Generalitat de Catalunya) 


\section{References}

1 Halliwell B. and Gutteridge J.M.C. (1999) Free Radicals in Biology and Medicine, $3^{\text {rd }}$ ed., Oxford University Press, Oxford

2 Frand A.R., Cuozzo, J.W. and Kaiser, C.A. (2000) Pathways for protein disulphide bond formation. Trends Cell Biol. 10, 203-210

3 Giles N.M., Watts A.B., Giles G.I., Fry F.H., Littlechild J.A. and Jacob C. (2003) Metal and redox modulation of cysteine protein function. Chem. Biol. 10, 677-693

4 Johnson D.C., Dean D.R., Smith A.D. and Johnson M.K. (2005) Structure, function and formation of biological iron-sulfur clusters. Annu. Rev. Biochem. 74, 247-281

5 Cabiscol E. and Ros J. (2006) Oxidative damage to proteins: structural modifications and consequences in cell function. In: Redox Proteomics, pp. 399471, Dalle-Donne, I., Scaloni, A. and Butterfield, D.A. (eds.), Wiley-Interscience, Hoboken, New Jersey

6 Biteau B., Labarre J. and Toledano M.B. (2003) ATP-dependent reduction of cysteine sulfinic acid by $S$. cerevisiae sulfiredoxin. Nature 425, 980-984

7 Cotgreave I.A. and Gerdes R.G. (1998) Recent trends in glutathione biochemistry-glutathione-protein interactions: a molecular link between oxidative stress and cell proliferation? Biochem. Biophys. Res. Commun. 242, 1-9

8 Biswas S., Chida A.S. and Rahman I. (2006) Redox modifications of proteinthiols. Emerging roles in cell signaling. Biochem. Pharmacol. 71, 551-564

9 Linke K. and Jakob U. (2003) Not every disulfide lasts forever: disulfide bond formation as a redox switch. Antiox. Red. Sign. 5, 425-434

10 Ghezzi P. (2005) Oxidoreduction of protein thiols in redox regulation. Biochem. Soc. Trans. 33, 1378-1381

11 Pineda-Molina E., Klatt P., Vázquez J., Marina A., García de Lacoba M., PérezSala D. and Lamas S. (2001) Glutathionylation of the p50 subunit of NF-kappaB: a mechanism for redox-induced inhibition of DNA binding. Biochemistry 40, 14134-14142

12 Klatt P., Molina E.P. and Lamas S. (1999) Nitric oxide inhibits c-Jun DNA binding by specifically targeted S-glutathionylation. J. Biol. Chem. 274, 15857-15864

13 Shenton D. and Grant C.M. (2003) Protein S-thiolation targets glycolysis and protein synthesis in response to oxidative stress in the yeast Saccharomyces cerevisiae. Biochem. J. 374, 513-519

14 Hoffman J.H., Linke K., Graf P.C., Lilie H. and Jakob U. (2004) Identification of a redox-regulated chaperone network. EMBO J. 23, 160-168 
15 Choi H., Kim S., Mukhopadhyay P., Cho S., Woo J., Storz G. and Ryu S. (2001) Structural basis of the redox switch in the OxyR transcription factor. Cell 105, 103-113

16 Holmgren A. (1989) Thioredoxin and glutaredoxin systems. J. Biol. Chem. 254, 13963-13966

17 Vlamis-Gardikas A. and Holmgren A. (2002) Thioredoxin and glutaredoxin isoforms. Methods Enzymol. 347, 286-296

18 Fernandes A.P. and Holmgren A. (2004) Glutaredoxins: glutathione-dependent redox enzymes with function as far beyond a simple thioredoxin bacjup system. Antiox. Red. Sign. 6, 63-74

19 Holmgren A., Johansson C., Berndt C., Lönn M.E., Hudemann C. and Lillig C.H. (2005) Thiol redox control via thioredoxin and glutaredoxin systems. Biochem. Soc. Trans. 33, 1375- 1377

20 Gelhaye E., Rouhier N., Navrot N. and Jacquot J.P. (2005) The plant thioredoxin systems. Cell. Mol. Life Sci. 62, 24-35

21 Rodríguez-Manzaneque M.T., Ros J., Cabiscol E., Sorribas A. and Herrero E. (1999) Grx5 glutaredoxin plays a central role in protection against oxidative damage in Saccharomyces cerevisiae. Mol. Cell. Biol. 19, 8180-8190

22 Vlamis-Gardikas A., Aslund F., Spyrou G., Bergman T. and Holmgren A. (1997) Cloning, overexpression and characterization of glutaredoxin 2, an atypical glutaredoxin from Escherichia coli. J. Biol. Chem. 272, 11236-11243

23 Lemaire S.D. (2004) The glutaredoxin family in oxygenic photosynthetic organisms. Photosynth. Res. 79, 305-318

24 Deponte M., Becker K. and Rahlfs S. (2005) Plasmodium falciparum glutaredoxin-like proteins. Biol. Chem. 386, 33-40

25 Rouhier N., Couturier J. and Jacquot J.P. (2006) Genome-wide analysis of plant glutaredoxin systems. J. Exp. Bot. 57, 1685-1696

26 Herrero E., Ros J., Tamarit J. and Bellí G. (2006) Glutaredoxins in fungi. Photosynth. Res. 89, 127-140

27 Wheeler G.L. and Grant CM. (2004) Regulation of redox homeostasis in the yeast Saccharomyces cerevisiae. Physiol. Plant. 120, 12-20

28 Holmgren A. (1976) Hydrogen donor system for Escherichia coli ribonucleosidediphosphate reductase dependent upon glutathione. Proc. Natl. Acad. Sci. USA 73, 2275-2279

29 Martin J.L. (1995) Thioredoxin- a fold for all reasons. Structure 3, 245-250

30 Hayes J.D., Flanagan J.U. and Jowsey I.R. (2005) Glutathione transferases. Annu. Rev. Pharmacol. Toxicol. 45, 51-88 
31 Holmgren A. and Aslund F. (1995) Glutaredoxin. Methods Enzymol. 252, 283-292

32 Peltoniemi M.J., Karala A.R, Jurvansuu J.K., Kinnula V.L. and Ruddock L.W. (2006) Insights into deglutathionylation reactions: different intermediates in the glutaredoxin and protein disulfide isomerase catalized reactions are defined by the $\gamma$-linkage present in glutathione. J. Biol. Chem. 281, 33107-33114

33 Bushweller J.H., Aslund F., Wuthrich K. and Holmgren A. (1992) Structural and functional characterization of the mutant Escherichia coli glutaredoxin (C14-S) and its mixed disulfide with glutathione. Biochemistry 31, 9288-9293

34 Rouhier N., Gelhaye E. and Jacquot J.P. (2004) Plant glutaredoxins: still mysterious reducing systems. Cell. Mol. Life Sci. 61, 1266-1277

35 Meyer Y., Riondet C., Constans L., Abdelgawwad M.R., Reichheld J.P. and Vignols F. (2006) Evolution of redoxin genes in the green lineage. Photosynth. Res. 89, 1-14

36 Carmel-Harel O. and Storz G. (2000) Roles of the glutathione- and thioredoxindependent reduction systems in the Escherichia coli and Saccharomyces cerevisiae responses to oxidative stress. Annu. Rev. Microbiol. 54, 439-461

37 Achebach S., Tran Q.H., Vlamis-Gardikas A., Müllner M., Holmgren A. and Unden G. (2004) Stimulation of Fe-S cluster insertion into apoFNR by Escherichia coli glutaredoxins 1, 2 and 3 in vitro. FEBS Lett. 565, 203-206

38 Luikenhuis S., Perrone G., Dawes I.W. and Grant C.M. (1998) The yeast Saccharomyces cerevisiae contains two glutaredoxin genes that are required for protection against reactive oxygen species. Mol. Biol. Cell 9, 1081-1091

39 Porras P., Padilla, C.A., Krayl M., Voos W. and Bárcena J.A. (2006) One single in-frame AUG codon is responsible for a diversity of subcellular localizations of glutaredoxin 2 in Saccharomyces cerevisiae. J. Biol. Chem. 281, 16551-16562

40 Collinson E.J., Wheeler G., Garrido E.O., Avery A.M., Avery S.V. and Grant C.M. (2002) The yeast glutaredoxins are active as glutathione peroxidases. J. Biol. Chem. 277, 16712-16717

41 Collinson E.J. and Grant C.M. (2003) Role of yeast glutaredoxins as glutathione S-transferases. J. Biol. Chem. 278, 22492-22497

42 Lee K.O., Lee J.R., Yoo J.Y. , Jang H.H., Moon J.C., Chi Y.H., Park S.K., Lee S.S., Lim C.O., Yun D.J., Cho M.J. and Lee S.Y. (2002) GSH-dependent peroxidase activity of the rice (Oryza sativa) glutaredoxin, a thiol transferase. Biochem. Biophys. Res. Commun. 296, 1152-1156

43 Mukhopadhyay R., Shi J. and Rosen B.P. (2000) Purification and characterization of Acr2p, the Saccharomyces cerevisiae arsenate reductase. J. Biol. Chem. 275, 21149-21157 
44 Gladyshev V.N., Liu A., Novoselov S.V., Krysan K., Sun Q.A., Kryukov V.M., Kryukov G.V. and Lou M.F. (2001) Identification and characterization of a new mammalian glutaredoxin (thioltransferase), Grx2. J. Biol. Chem. 276, 3037430380

45 Lundberg M., Johanson C., Chandra J., Enoksson M., Jacobsson G., Ljung J., Johansson M. and Holmgren A. (2001) Cloning and expression of a novel human glutaredoxin (Grx2) with mitochondrial and nuclear isoforms. J. Biol. Chem. 276, 26269-26275

46 Lillig C.H., Berndt C., Vergnolle O., Lönn M.E., Hudemann C., Bill E. and Holmgren A. (2005) Characterization of human glutaredoxin 2 as iron-sulfur protein: a possible role as redox sensor. Proc. Natl. Acad. Sci. USA 102, 81688173

47 Johansson C., Kavanagh K.L., Gileadi O. and Oppermann U. (2007) Reversible sequestration of active site cysteines in a 2Fe2S-bridged dimmer provides a mechanism for glutaredoxin 2 regulation in human mitochondria. J. Biol. Chem. Doi10.1074/jbc.M608179200

48 Feng Y., Zhong N., Rouhier N., Hase T., Kusunoki M., Jacquot J.P., Jin C. and Xia B. (2006) Structural insight into poplar glutaredoxin C1 with a bridging ironsulfur cluster at the active site. Biochemistry 45, 7998-8008

49 Carroll M.C., Outten C., Proescher J.B., Rosenfeld L., Watson W.H., Whitson L.J., Hert P.J., Jensen L.T. and Culotta V.C. (2006) The effects of glutaredoxin and copper activation pathways on the disulfide and stability of $\mathrm{Cu} / \mathrm{Zn}$ superoxide dismutase. J. Biol. Chem 281, 28648-28656

50 Bellí G., Polaina J., Tamarit J., de la Torre M.A., Rodríguez-Manzaneque M.T., Ros J. and Herrero E. (2002) Structure-function analysis of yeast Grx5 monothiol gluteredoxin defines essential amino acids for the function of the protein. J. Biol. Chem. 277, 37590-37596

51 Vilella F., Alves R., Rodríguez-Manzaneque M.T., Bellí G., Swaminathan S., Sunnerhagen P. and Herrero E. (2004) Evolution and cellular function of monothiol glutaredoxins: involvement in iron-sulfur cluster assembly. Comp. Funct. Genom. 5, 328-341

52 Molina M.M., Bellí G., de la Torre M.A., Rodríguez-Manzaneque M.T. and Herrero E. (2004) Nuclear monothiol glutaredoxins of Saccharomyces cerevisiae can function as mitochondrial glutaredoxins. J. Biol. Chem. 279, 51923-51930

53 Powis G. and Montfort W.R. (2001) Properties and biological activities of thioredoxins. Annu. Rev. Pharmacol. Toxicol. 41, 261-295 
54 Molina-Navarro M.M., Casas C., Piedrafita L., Bellí G. and Herrero E. (2006) Prokaryotic and eukaryotic monothiol glutaredoxins are able to perform the functions of Grx5 in the biogenesis of Fe/S clusters in yeast mitochondria. FEBS Lett. 580, 2273-2280

55 Isakov N., Witte S. and Altman A. (2000) PICOT-HD: a highly conserved protein domain that is often associated with thioredoxin and glutaredoxin modules. Trends Biochem. Sci. 25, 537-539

56 Fladvad M., Bellanda M., Fernandes A.P., Mammi S., Vlamis-Gardikas A., Holmgren A. and Sunnerhagen M. (2005) Molecular mapping of functionalities in the solution structure of reduced Grx4, a monothiol glutaredoxin from Escherichia coli. J. Biol. Chem. 280, 24553-24561

57 Fernandes A.P., Fladvad M., Berndt C., Andrésen C., Lillig C.H., Neubauer P., Sunnerhagen M., Holmgren A. and Vlamis-Gardikas A. (2005) A novel monothiol glutaredoxin ( $\mathrm{Gr} \times 4)$ from Escherichia coli can serve as a substrate for thioredoxin reductase. J. Biol. Chem. 280, 24544-24552

58 Tamarit J., Bellí G., Cabiscol E., Herrero E. and Ros J. (2003) Biochemical characterization of yeast mitochondrial Grx5 monothiol glutaredoxin. J. Biol. Chem. 278, 25745-25751

59 Rahlfs S., Fischer M. and Becker K. (2001) Plasmodium falciparum possesses a classical glutaredoxin and a second, glutaredoxin-like protein with a PICOT homology domain. J. Biol. Chem. 276, 37133-37140

60 Herrero E. and Ros J. (2002) Glutaredoxins and oxidative stress defence in yeast. Methods Enzymol. 348, 136-146

61 Rodríguez-Manzaneque M.T., Tamarit J., Bellí G., Ros J. and Herrero E. (2002) Grx5 is a mitochondrial glutaredoxin required for the activity of iron/sulfur enzymes. Mol. Biol. Cell 13, 1109-1121

62 Chung W.H., Kim K.D. and Roe J.H. (2005) Localization and function of three monothiol glutaredoxins in Schizosaccharomyces pombe. Biochem. Biophys. Res. Commun. 330, 604-610

63 Cheng N.H., Liu J.Z., Brock A., Nelson R.S. and Hirschi K.D. (2006) AtGRXcp, an Arabidopsis chloroplastic/plastidic glutaredoxin is critical for protection against protein oxidative damage. J. Biol. Chem. 281, 26280-26288

64 Lopreiato R., Facchin S., Sartori G., Arrigoni G., Casonato S., Ruzzene M., Pinna L.A. and Carignani G. (2004) Analysis of the interaction between piD261/Bud32, an evolutionary conserved protein kinase of Saccharomyces cerevisiae, and the Grx4 glutaredoxin. Biochem. J. 377, 395-405 
65 Babichev Y. and Isakov N. (2001) Tyrosine phosphorylation of PICOT and its translocation to the nucleus in response of human T cells to oxidative stress. Adv. Exp. Med. Biol. 495, 41-45

66 Scherens B. and Goffeau A. (2004) The uses of genome-wide yeast mutant collections. Genome Biol. 5, 229

67 Lill R. and Mühlenhoff U. (2005) Iron-sulfur-protein biogenesis in eukaryotes. Trends Biochem. Sci. 30, 133-141

68 Shenton D., Perrone G., Quinn K.A., Dawes I.W. and Grant C.M. (2002) Regulation of protein S-thiolation by glutaredoxin 5 in the yeast Saccharomyces cerevisiae. J. Biol. Chem. 277, 16853-16859

69 Rouault T.A. and Tong W.H. (2005) Iron-sulfur cluster biogenesis and mitochondrial iron homeostasis. Nat. Rev. Mol. Cell Biol. 6, 345-351

70 Lill R., Dutkiewicz R., Elsässer H.P., Hausmann A., Netz D.J.A., Pierik A.J., Stehling O., Urzica E. and Mühlenhoff U. (2006) Mechanisms of iron-sulfur protein maturation in mitochondria, cytosol and nucleus of eukaryotes. Biochem. Biophys. Acta 1763, 652-667

71 Lill R. and Mühlenhoff U. (2006) Iron-sulfur protein biogenesis in eukaryotes: components and mechanisms. Annu. Rev. Cell Dev. Biol 22, 457-486

72 Rutherford J.C., Ojeda L., Balk J., Mühlenhoff U., Lill R. and Winge D.R. (2005) Activation of the iron regulon by the yeast Aft1/Aft2 transcription factors depends on mitochondrial but not cytosolic iron-sulfur protein biogenesis. J. Biol. Chem. 280, 10135-10140

73 Yamaguchi-Iwai Y., Dancis A. and Klausner R.D. (1995) AFT1: a mediator of iron regulated transcriptional control in Saccharomyces cerevisiae. EMBO J. 14, 1231-1239

74 Casas C., Aldea M., Espinet C., Gallego C., Gil R. and Herrero E. (1997) The Aft1 transcription factor is differentially required for expression of high-affinity iron uptake genes in Saccharomyces cerevisiae. Yeast 13, 621-637

75 Blaiseau P.L., Lesuisse E. and Camadro J.M. (2001) Aft2p, a novel iron-regulated transcriptional activator that modulates, with Aft1p, intracellular iron use and resistance to oxidative stress in yeast. J. Biol. Chem. 276, 34221-34226

76 Rutherford J.C., Jaron S., Ray E., Brown P.O. and Winge D.R. (2001) A second iron-regulatory system in yeast independent of Aft1p. Proc. Natl. Acad. Sci. USA 98, 14322-14327

77 Bellí G., Molina M.M., García-Martínez J., Pérez-Ortín J.E. and Herrero E. (2004) Saccharomyces cerevisiae glutaredoxin 5-deficient cells subjected to continuous 
oxidizing conditions are affected in the expression of specific sets of genes. J. Biol. Chem. 279, 12386-12395

78 Foury F. and Talibi D. (2001) Mitochondrial control of iron homeostasis: a genome-wide analysis of gene expression in a yeast frataxin deficient strain. J. Biol. Chem. 276, 7762-7768

79 Chen O.S., Crisp R.J., Valachovic M., Bard M., Winge D.R. and Kaplan J. (2004) Transcription of the yeast iron regulon does not respond directly to iron but rather to iron-sulfur cluster biosynthesis. J. Biol. Chem. 279, 29513-29518

80 Irazusta V., Cabiscol E., Reverter-Branchart G., Ros J. and Tamarit J. (2006) Manganese is the link between frataxin and iron-sulfur deficiency in the yeast model of Friedreich ataxia. J. Biol. Chem. 281, 12227-12232

81 Yang M., Cobine P.A., Molik S., Naranuntarat A., Lill R., Winge D.R. and Culotta V.C. (2006) The effectors of mitochondrial iron homeostasis on cofactor specificity of superoxide dismutase 2. EMBO J. 25, 1775-1783

82 Mühlenhoff U., Gerber J., Richhardt N. and Lill R. (2003) Components involved in assembly and dislocation of iron-sulfur clusters on the scaffold protein Isu1p. EMBO J. 22, 4815-4825

83 Ding H., Harrison K. and Lu J. (2005) Thioredoxin reductase system mediates iron binding in IscA and iron delivery for the iron-sulfur cluster assembly in IscU. J. Biol. Chem. 280, 30432-30437

84 Alves R., Herrero E. and Sorribas A. (2004) Predictive reconstruction of the mitochondrial iron-sulfur cluster assembly metabolism. II. Role of glutaredoxin Grx5. Proteins 57, 481-492

85 Kim H.G., Park E.H. and Lim C.J. (2005) The fission yeast gene encoding monothiol glutaredoxin 5 is regulated by nitrosative and osmotic stresses. Mbl. Cells 20, 43-50

86 Song J.Y., Cha J., Lee J. and Roe J.H. (2006) Glutathione reductase and a mitochondrial thioredoxin play overlapping roles in maintaining iron-sulfur enzymes in fission yeast. Eukaryot. Cell 5, 1857-1865

87 Gerdes S.Y., Scholle M.D., Campbell J.W., Balazsi G., Ravasz E., Daugherty M.D., Somera A.L., Kyrpides N.C., Anderson I., Gelfand M.S., Bhattacharya A., Kapatral V., D'Souza M., Baev M.V., Grechkin Y., Mseeh F., Fonstein M.Y., Overbeek R., Barabasi A.L., Oltvai Z.N. and Osterman A.L. (2003) Experimental determination and system level analysis of essential genes in Escherichia coli MG1655. J. Bacteriol. 185, 5673-5684

88 Wingert R.A., Galloway J.L., Barut B., Foott H., Fraenkel P., Axe J.L., Weber G.J., Dooley K., Davidson A.J., Schmidt B., Paw B.H., Shaw G.C., Kingsley P., 
Palis J., Schubert H., Chen O., Kaplan J., the Tübingen 2000 Screen Consortium and Zon L.I. (2005) Deficiency of glutaredoxin 5 reveals Fe-S clusters are required for vertebrate haem synthesis. Nature 436, 1035-1039

89 Pujol-Carrión, N., Belli, G., Herrero, E., Bogues, A. and de la Torre-Ruiz, M.A. (2006) Glutaredoxins Grx3 and Grx4 regulate nuclear localisation of Aft1 and the oxidative stress response in Saccharomyces cerevisiae. J. Cell Sci. 119, 45544564

90 Yamaguchi-Iwai, Y., Ueta, R., Fukunaka, A. and Sasaki, R. (2002) Subcellular localisation of Aft1 transcription factor responds to iron status in Saccharomyces cerevisiae. J. Biol. Chem. 277, 18914-18918

91 Ojeda, L., Keller, G., Muhlenhoff, U., Rutherford, J.C., Lill, R. and Winge, D.R. (2006) Role of glutaredoxin-3 and glutaredoxin-4 in the iron-regulation of the Aft1 transcriptional activator in Saccharomyces cerevisiae. J. Biol. Chem. 281, 1766117669

$92 \mathrm{Ni}$, L. and Snyder, M. (2001) A genomic study of the bipolar bud site selection pattern in Saccharomyces cerevisiae. Mol. Biol. Cell 12, 2147-2170

93 Downey, M., Houlsworth, R., Maringele, L., Rollie, A., Brehme, M., Galicia, S., Guillard, S., Partington, M., Zubko, M.K., Krogan, N.J., Emili, A., Greenblatt, J.F., Harrington, L., Lydall, D. and Durocher, D. (2006) A genome-wide screen identifies the evolutionarily conserved KEOPS complex as a telomere regulator. Cell 124, 1155-1168

94 Kisseleva-Romanova, E., Lopreiato, R., Baudin-Baillieu, A., Rousselle, J.C., Ilan, L., Hofmann, K., Namane, A., Mann, C. and Libri, D. (2006) Yeast homolog of a cancer-testis antigen defines a new transcription complex. EMBO $\mathrm{J} 25$, 35763585

95 Moon, J.S., Lim, H.W., Park, E.H. and Lim, C.J. (2005) Characterization and regulation of the gene encoding monothiol glutaredoxin 3 in the fission yeast Schizosaccharomyces pombe. Mol. Cells 20, 74-82

96 Witte, S., Villalba, M., Bi, K., Liu, Y., Isakov, N. and Altman, A. (2000) Inhibition of the c-Jun N-terminal kinase/AP-1 and NF-kappaB pathways by PICOT, a novel protein kinase C-interaction protein with a thioredoxin homology domain. J. Biol. Chem. 275, 1902-1909

97 Jeong, D., Cha, H., Kim, E., Kang, M., Yang, D.K., Kim, J.M., Yoon, P.O., Oh, J.G., Bernecker, O.Y., Sakata, S., Le, T.T., Cui, L., Lee, Y.H., Kim do, H., Woo, S.H., Liao, R., Hajjar, R.J. and Park, W.J. (2006) PICOT inhibits cardiac hypertrophy and enhances ventricular function and cardiomyocyte contractility. Circ. Res. 99, 307-314 
98 Heidkamp, M.C., Bayer, A.L., Martin, J.L. and Samarel, A.M. (2001) Differential activation of mitogen-activated protein kinase cascades and apoptosis by protein kinase $C-\varepsilon$ and $-\delta$ in neonatal ventricular myocites. Circ. Res. 89, 882-890

99 Levin D.E. (2005) Cell wall integrity signalling in Saccharomyces cerevisiae. Microbiol. Mol. Biol. Rev. 69, 262-291

100 Sheehan, D., Meade, G., Foley, V.M. and Dowd, C.A. (2001) Structure, function and evolution of glutathione transferases: implications for classification of nonmammalian members of an ancient enzyme superfamily. Biochem. J. 360, 1-16

101 Board, P.G., Coggan, M., Chelvanagayam, G., Easteal, S., Jermiin, L.S., Schulte, G.K., Danley, D.E., Hoth, L.R., Griffor, M.C., Kamath, A.V., Rosner, M.H., Chrunyk, B.A., Perregaux, D.E., Gabel, C.A., Geoghegan, K.F. and Pandit, J. (2000) Identification, characterization, and crystal structure of the omega class glutathione transferases. J. Biol. Chem. 275, 24800-24806

102 Whitbread, A.K., Masoumi, A., Tetlow, N., Schmuck, E., Coggan, M. and Board, P.G. (2005) Characterization of the Omega-class of glutathione transferases. Methods Enzymol. 401, 77-99

103 Garcerá, A., Barreto, L., Piedrafita, L., Tamarit, J. and Herrero, E. (2006) Saccharomyces cerevisiae cells have three Omega-class glutathione transferases acting as 1-Cys thiol transferases. Biochem. J. 398, 187-196

104 Barreto, L., Garcerá, A., Jansson, K., Sunnerhagen, P. and Herrero, E. (2006) A peroxisomal glutathione transferase of Saccharomyces cerevisiae is functionally related to sulfur amino acid metabolism. Eukaryot. Cell 5, 1748-1759

105 Gabaldón, T., Snel, B., Zimmeren, F., Hemrika, W., Tabak, H. and Huynen, M.A. (2006) Origin and evolution of the peroxisomal proteome. Biol. Direct 1, 8 


\section{Figure legends}

Figure 1. (A) Components of the glutaredoxin system in the reduction of dithiol groups. These can consist of protein disulphides or of mixed disulphides between a protein and a glutathione molecule. GRX, glutaredoxin; GSH, reduced glutathione; GSSG, glutathione disulphide. (B) Dithiol and monothiol mechanisms of action of glutaredoxins for reduction of dithiol groups. Active cysteine residues in glutaredoxin molecules are indicated as $\mathrm{SH}$ or $\mathrm{S}^{-}$depending on the redox state. See text for details.

Figure 2. Structure of monothiol glutaredoxins from different organisms. The position and size of mitochondrial/plastid targeting sequences (blue boxes) and glutaredoxin (red) and thioredoxin-like (blue) domains is ndicated. Numbers correspond to the position of the cysteine residue in the active site CGFS motif and of a partially conserved second C-terminal cysteine and to the total length of the molecules. These are named according to the more recent literature.

Figure 3. Summary of experimentally determined functions for monothiol glutaredoxins, with the organisms in which these functions have been demonstrated. 
(A)

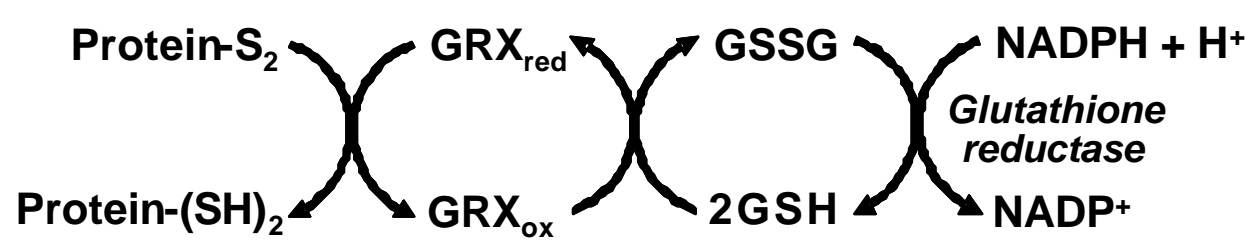

(B)
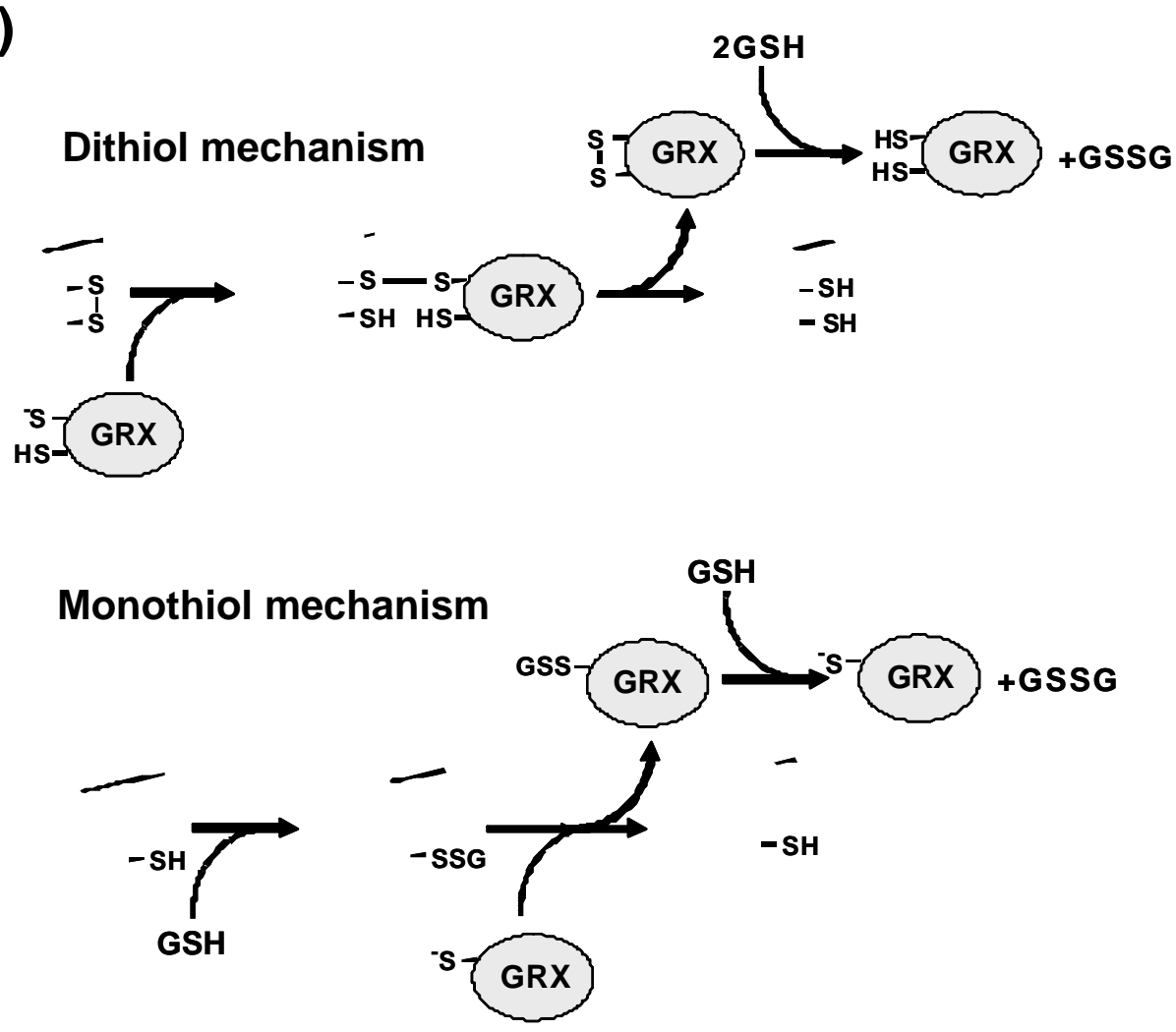

Figure 1 


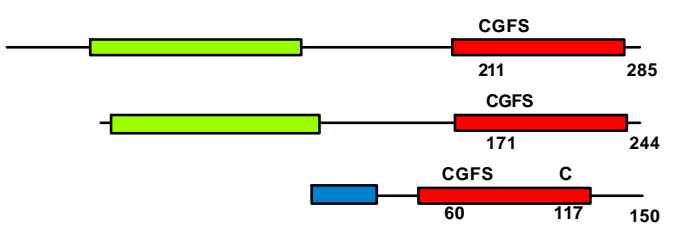

\begin{tabular}{lll} 
CGFS & \multicolumn{1}{c}{$C$} & \\
\hline 30 & 84 & 115
\end{tabular}
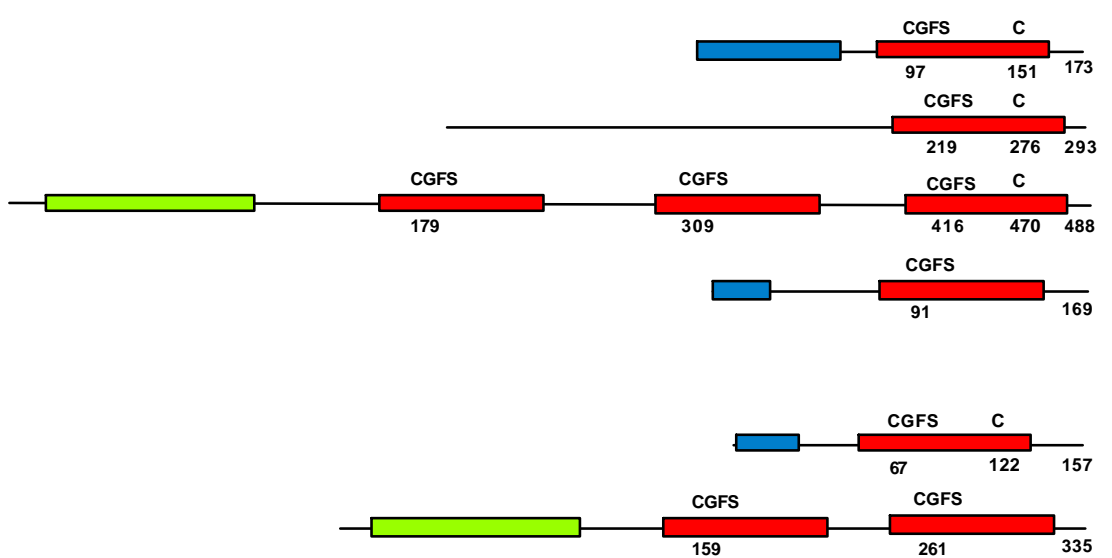

S. cerevisiae Grx3

Grx4

Grx5

E. coli Grx4

A. thaliana AtGRXcp

AtGRX2

AtGRX3

AtGRX4

Human hGRX5

PICOT

Figure 2 


\section{Regulation of protein} kinase $\mathrm{C}$ activity

MAMMALS
Synthesis of iron/sulphur clusters in mitochondria
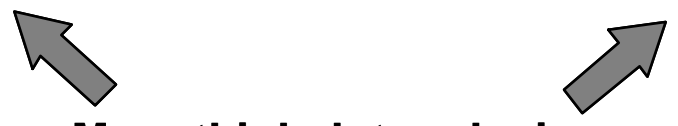

EUKARYOTES(AND

BACTERIA?)

Monothiol glutaredoxins

(CGFS motif)

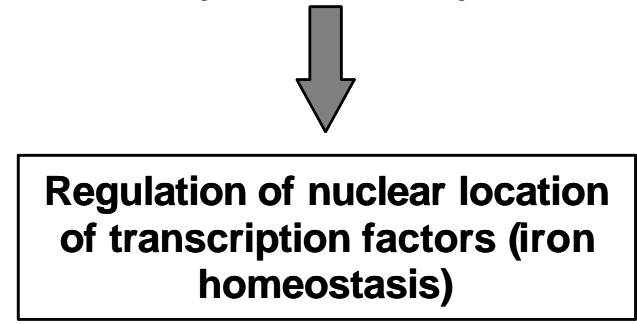

YEAST

Figure 3 